\title{
Correlated hopping of electrons: Effect on the Brinkman-Rice transition and the stability of metallic ferromagnetism
}

\author{
M. Kollar* and D. Vollhardt \\ Theoretical Physics III, Center for Electronic Correlations and Magnetism, Institute of Physics, \\ University of Augsburg, D-86135 Augsburg, Germany \\ (Received 2 August 2000; published 8 January 2001)
}

\begin{abstract}
We study the Hubbard model with bond-charge interaction ("correlated hopping") in terms of the Gutzwiller wave function. We show how to express the Gutzwiller expectation value of the bond-charge interaction in terms of the correlated momentum-space occupation. This relation is valid in all spatial dimensions. We find that in infinite dimensions, where the Gutzwiller approximation becomes exact, the bond-charge interaction lowers the critical Hubbard interaction for the Brinkman-Rice metal-insulator transition. The bondcharge interaction also favors ferromagnetic transitions, especially if the density of states is not symmetric and has a large spectral weight below the Fermi energy.
\end{abstract}

DOI: $10.1103 /$ PhysRevB.63.045107

PACS number(s): 71.27.+a

The microscopic origin of magnetic ordering in systems like transition metals, transition-metal oxides, and hightemperature superconductors is intricate, since it is due to correlations between the electrons. The simplest model to attempt a description of such systems is the single-band Hubbard model ${ }^{1-3}$

$$
\hat{H}_{\text {Hubbard }}=\sum_{i j \sigma} t_{i j} \hat{c}_{i \sigma}^{+} \hat{c}_{j \sigma}^{+}+U \sum_{i} \hat{n}_{i \uparrow} \hat{n}_{i \downarrow}
$$

where hats indicate operators. This model describes the competition between kinetic and potential energy which is at the heart of the quantum-mechanical correlation problem. ${ }^{4}$ The Hubbard interaction $U$ represents the Coulomb repulsion of electrons in the same orbital at a given lattice site. It is given by the matrix element $U=\left\langle i i\left|V\left(\boldsymbol{r}-\boldsymbol{r}^{\prime}\right)\right| i i\right\rangle$ of the Coulomb potential, and is typically on the order of a few eV. The matrix elements involving neighboring lattice sites $i$ and $j$ are generally smaller than $U$, but may not be negligibly small. One of them is the bond-charge interaction $X_{i j}=\langle i i| V(\boldsymbol{r}$ $\left.-\boldsymbol{r}^{\prime}\right)|i j\rangle,{ }^{1,5-7}$ which is typically on the order of $0.1-1 \mathrm{eV}$ and hence is comparable in magnitude to the tight-binding hopping amplitude $t_{i j}$. It describes a density-dependent hopping of the electrons

$$
\hat{H}_{X}=\sum_{i j \sigma} X_{i j} \hat{c}_{i \sigma}^{+} \hat{c}_{j \sigma}^{+}\left(\hat{n}_{i \bar{\sigma}}+\hat{n}_{j \bar{\sigma}}\right)
$$

which only contributes if the lattice site from or onto which an electron with spin $\sigma$ is hopping is occupied by an electron with spin $\bar{\sigma}$ ("correlated hopping'"). The effect of $\hat{H}_{X}$ competes strongly with both the kinetic energy and the Hubbard interaction. Correlated hopping of spin- $\sigma$ electrons between two sites is enhanced if spin- $\bar{\sigma}$ electrons are present, but this in turn will cost the latter kinetic energy, as well as Coulomb energy for the double occupations. Moreover, in a band picture the coupling of densities and kinetic energy can lead to a band narrowing which lowers the amount of energy that is necessary for a ferromagnetic spin polarization. These mechanisms explain why, in principle, $\hat{H}_{X}$ may play an important role in the stabilization of ferromagnetism and the localization of electrons.

The model $\hat{H}=\hat{H}_{\text {Hubbard }}+\hat{H}_{X}$ cannot be solved exactly, and only mean-field and finite-size diagonalization results are available. ${ }^{8}$ Recently Schiller ${ }^{9}$ showed how to incorporate $\hat{H}_{X}$ into the framework of dynamical mean-field theory, ${ }^{10,11}$ but no numerical results have yet been obtained for $\hat{H}$.

One of the standard tools to approach the correlated electron problem is the Gutzwiller wave function ${ }^{3}$

$$
\left|\Psi_{G}\right\rangle=\prod_{i}\left[1-(1-g) \hat{n}_{i \uparrow} \hat{n}_{i \downarrow}\right]\left|\Phi_{0}\right\rangle
$$

where $g$ is a variational parameter $(0 \leqslant g \leqslant 1)$, and the starting wave function $\left|\Phi_{0}\right\rangle$ is a product state of spin-up and spin-down Fermi seas. By construction both $\left|\Phi_{0}\right\rangle$ and $\left|\Psi_{G}\right\rangle$ are translationally invariant and have a fixed particle density $n=n_{\uparrow}+n_{\downarrow}$ and magnetization $m=n_{\uparrow}-n_{\downarrow}$. The uncorrelated case $U=X_{i j}=0$ corresponds to $g=1$, while $U=\infty$ forbids any doubly occupied sites and thus corresponds to $g=0$. For $m \neq 0$ and $g \neq 0$ the wave function $\left|\Psi_{G}\right\rangle$ describes an itinerant ferromagnetic state. Starting wave functions $\left|\Phi_{0}\right\rangle$ with other broken symmetries can also be considered. Here, however, we will only consider paramagnetism and ferromagnetism.

Using the Gutzwiller wave function one may, in principle, calculate expectation values of any operator $\hat{A}$ as $\langle\hat{A}\rangle_{G}$ $=\left\langle\Psi_{G}|\hat{A}| \Psi_{G}\right\rangle /\left\langle\Psi_{G} \mid \Psi_{G}\right\rangle$. The energy expectation value $E$ $=\langle\hat{H}\rangle_{G}$, when optimized with respect to $g$, is an upper bound for the exact ground-state energy of $\hat{H}$ by the variational principle. The variational energy $E$ can be written as

$$
E=\langle\hat{H}\rangle_{G}=\sum_{k \sigma} \epsilon_{k} n_{k \sigma}+\sum_{k \sigma} 2 \xi_{k} \operatorname{Re}\left(x_{k \sigma}\right)+U d,
$$

where the $k$-space occupation $n_{k \sigma}$, bond-charge occupation $x_{k \sigma}$, and double occupation $d$ are defined by ( $L$ is the number of lattice sites) 


$$
\begin{gathered}
n_{\boldsymbol{k} \sigma}=\frac{1}{L} \sum_{i \neq j} e^{i \boldsymbol{k}\left(\boldsymbol{R}_{i}-\boldsymbol{R}_{j}\right)}\left\langle\hat{c}_{i \sigma}^{+} \hat{c}_{j \sigma}^{+}\right\rangle_{G}, \\
x_{\boldsymbol{k} \sigma}=\frac{1}{L} \sum_{i \neq j} e^{i \boldsymbol{k}\left(\boldsymbol{R}_{i}-\boldsymbol{R}_{j}\right)}\left\langle\hat{n}_{i \bar{\sigma}} \hat{c}_{i \sigma}^{+} \hat{c}_{j \sigma}^{+}\right\rangle_{G}, \\
d=\frac{1}{L} \sum_{i}\left\langle\hat{n}_{i \uparrow} \hat{n}_{i \downarrow}\right\rangle_{G} .
\end{gathered}
$$

They each depend on $g, n$, and $m$. Here $\epsilon_{k}$ and $\xi_{k}$ are the Fourier transforms of $t_{i j}$ and $X_{i j}$, respectively. By convention, $t_{i i}=X_{i i}=0$.

In general the expectation values in Eqs. (5)-(7) are not independent of one another. In particular, for the Gutzwiller wave function the $k$ space and bond-charge occupation are closely related. Using the techniques of Ref. 12 we obtain, for all Bravais lattices in arbitrary dimensions $D$,

$$
x_{k \sigma}=\frac{\left[(1+g) n_{k \sigma}^{0}-g\right] n_{k \sigma}-n_{k \sigma}^{0}}{1-g}+n_{\sigma}^{-} n_{k \sigma}^{0}-d,
$$

with $n_{\boldsymbol{k} \sigma}^{0}=\left.n_{\boldsymbol{k} \sigma}\right|_{g=1}$ as the uncorrelated Fermi function. It should be noted that although $x_{k \sigma}$ and $n_{k \sigma}$ are linearly related, the bond-charge energy and the kinetic energy will generally not be, since the regions of the Brillouin zone inside $\left(n_{k \sigma}^{0}=1\right)$ and outside $\left(n_{k \sigma}^{0}=0\right)$ of the Fermi sea contribute differently.

Gutzwiller approximation. Now we turn to evaluate the variational energy $E$ within the Gutzwiller approximation, which is known to yield the exact evaluation of expectation values in terms of the Gutzwiller wave function in the limit of infinite spatial dimensions $(D \rightarrow \infty) \cdot{ }^{10,12-14}$ It describes a Fermi liquid $^{15}$ with piecewise constant $k$-space occupation,

$$
n_{k \sigma}=n_{\sigma}+\left(n_{k \sigma}^{0}-n_{\sigma}\right) q_{\sigma},
$$

where the discontinuity at the Fermi surface is given by

$$
q_{\sigma}=\frac{\left[\sqrt{\left(n_{\sigma}-d\right)(1-n+d)}+\sqrt{\left(n_{\sigma}^{-}-d\right) d}\right]^{2}}{n_{\sigma}\left(1-n_{\sigma}\right)} .
$$

The variational parameter $g$ is related to the double occupation $d$ by

$$
g^{2}=\frac{\left(n_{\uparrow}-d\right)\left(n_{\downarrow}-d\right)}{(1-n+d) d},
$$

and it is convenient to use the latter as variational parameter. For the variational energy we obtain

$$
E=E^{0}-\sum_{\sigma}\left(1-q_{\sigma}\right)\left(\epsilon_{0 \sigma}+\xi_{0 \sigma}\right)+U_{\mathrm{eff}}\left(d-n_{\uparrow} n_{\downarrow}\right),
$$

where $\epsilon_{0 \sigma}=(1 / L) \Sigma_{k} \epsilon_{k} n_{k \sigma}^{0}$ is the uncorrelated kinetic energy, and similarly $\xi_{0 \sigma}=(1 / L) \Sigma_{k} \xi_{k} n_{k \sigma}^{0}$. The effective Hubbard interaction $U_{\text {eff }}$ and the uncorrelated variational energy $E^{0}$ $\left(=\left.E\right|_{g=1}\right)$ in Eq. (12) are given by

$$
U_{\mathrm{eff}}=U+\sum_{\sigma} \frac{\left(1-2 n_{\sigma}\right)}{\left(1-n_{\sigma}\right) n_{\sigma}} \xi_{0 \sigma}
$$

$$
E^{0}=\sum_{\sigma}\left(\epsilon_{0 \sigma}+2 n_{\sigma}^{-} \xi_{0 \sigma}\right)+U n_{\uparrow} n_{\downarrow}
$$

Within the Gutzwiller approximation the bond-charge interaction thus leaves the form of the variational energy unchanged, but enters into the effective kinetic energy and effective Hubbard interaction via $\xi_{0 \sigma}$. The effect on the kinetic energy can be interpreted as a spin-dependent band narrowing or widening, which is also found in the HartreeFock approximation of the correlated hopping term, and can lead to a stabilization of ferromagnetism. ${ }^{6,8}$ However, the Gutzwiller approximation reveals two distinct effects that cannot be resolved in ordinary Hartree-Fock theory, where the suppression of double occupancies can only be achieved by spin polarizing the system. On the one hand, the kinetic energy increases if $\xi_{0 \sigma}>0$, i.e., the effective hopping becomes smaller. This corresponds to an effective narrowing of the band, which lowers the amount of energy that must be expended for a ferromagnetic spin polarization. Furthermore the bond-charge interaction contributes to $U_{\text {eff }}$, which suppresses double occupancies already at lower values of the bare Hubbard interaction $U$. The balance between these two effects is determined by the optimal variational parameter $d$.

Brinkman-Rice metal-insulator transition. We now discuss the effect of $\hat{H}_{X}$ on the Brinkman-Rice transition that occurs in the Gutzwiller approximation at half-filling ( $n$ $=1) \cdot{ }^{16,15}$ For convenience we define the strength of the bond-charge interaction $X$ by

$$
\xi_{0 \sigma}=-X \epsilon_{0 \sigma}
$$

Note that $\xi_{0 \sigma}$ will remain proportional to $\epsilon_{0 \sigma}$ according to Eq. (15) for all densities if $t_{i j}$ and $X_{i j}$ have the same range, e.g., if they are nonzero only for nearest-neighbor sites. In the limit of $D \rightarrow \infty$ both $t_{i j}$ and $X_{i j}$ must both be scaled as $1 / \sqrt{Z_{i j}}\left(Z_{i j}\right.$ is the number of neighbors $\left.i j\right),{ }^{10}$ which is compatible with Eq. (15). The dispersion $\epsilon_{k}$ enters only through the density of states (DOS) $N(\epsilon)$ (which determines $\epsilon_{0 \sigma}$ ), as expected in dimension $D=\infty$. We will consider several densities of states below.

With the above definition of $X$, the Gutzwiller approximation energy for $n=1$ and $m=0$ simplifies to $E=8 d(1$ $-2 d)(1-X) \epsilon_{0}+U d$, where $\epsilon_{0} \equiv \Sigma_{\sigma} \epsilon_{0 \sigma}<0$. Optimization with respect to $d$ yields a critical value for $U$,

$$
U_{c}(X)=8\left|\epsilon_{0}\right|(1-X),
$$

above which the localized state with $d=0$ is lowest in energy. Hence the Brinkman-Rice transition is moved to lower $U$ for $X>0$, i.e., the bond-charge interaction favors localization. (Only $U \geqslant 0$ and $X \leqslant 1$ will be considered from now on.) We find that the $U$ dependence of the double occupation $d$, the discontinuity of the $k$-space occupation $q\left(\equiv q_{\sigma}\right)$, and the energy $E$ is formally the same as in the original Brinkman-Rice theory for $X=0$, i.e.,

$$
\begin{gathered}
d=\frac{1}{4}\left(1-\frac{U}{U_{c}}\right), \\
q=1-\frac{U^{2}}{U_{c}^{2}},
\end{gathered}
$$




$$
E=-\frac{U_{c}}{8}\left(1-\frac{U^{2}}{U_{c}}\right)
$$

except that $U_{c}$ now depends on $X$ [Eq. (16)]. We note that the simultaneous vanishing of $q$ and double occupation $d$ at a finite value of $U$ is characteristic of the Brinkman-Rice transition, in contrast to the numerical solution of the Hubbard model, where $d$ remains nonzero across the transition. ${ }^{11,17}$

Ferromagnetic transition. The instability of the paramagnetic state toward ferromagnetism can be determined from the bulk susceptibility $\chi$. For half-filling, we obtain

$\frac{1}{\chi}=\frac{q}{2 N\left(\epsilon_{F}\right)}\left[(1-X)\left(1-\frac{p U\left(U+2 U_{\mathrm{c}}\right)}{\left(U+U_{\mathrm{c}}\right)^{2}}\right)+\frac{r X U_{\mathrm{c}}}{U+U_{\mathrm{c}}}\right]$,

where we have introduced the dimensionless parameters

$$
\begin{gathered}
p=4 N\left(\epsilon_{F}\right)\left|\epsilon_{0}\right|, \\
r=4 N\left(\epsilon_{F}\right) \epsilon_{F} .
\end{gathered}
$$

The Fermi energy $\epsilon_{F}$ in Eq. (22) represents an absolute scale since the first moment of the DOS is fixed at zero (due to $\left.t_{i i}=0\right)$. There are two factors in $\chi$ that can diverge: either $q \rightarrow 0$, i.e., the effective band mass $m^{*} / m=q^{-1}$ diverges at $U_{\mathrm{c}}(X)$, indicating a localization transition, or the Stoner-type factor in square brackets in Eq. (20) vanishes at

$U_{\mathrm{fm}}(X)=\frac{\sqrt{r^{2} X^{2}-4 p(1-p)(1-X)^{2}}-r X}{2(1-p)}+X-1$,

signaling an instability toward ferromagnetism. The latter instability precedes the localization transition whenever $p$ $>p_{\mathrm{fm}}$, where

$$
p_{\mathrm{fm}}=\frac{4}{3}\left(1+\frac{r X}{2(1-X)}\right) .
$$

These results reduce to the known values $p_{\mathrm{fm}}=\frac{4}{3}$ and $U_{\mathrm{fm}}$ $=(\sqrt{[p /(p-1)]}-1) U_{c}$ for $X=0 .^{16,15}$

Let us first consider the effect of the bond-charge interaction in the case of a symmetric DOS, $N(\epsilon)=N(-\epsilon)$, which results if hopping takes place only between different sublattices of a bipartite lattice. The Fermi energy at half-filling is then $\epsilon_{F}=0$; hence $r=0$. In this case $p_{\mathrm{fm}}=\frac{4}{3}$, the same criterion as for $X=0$. On the other hand, for an asymmetric DOS ferromagnetism is favored by $X>0$ if $r<0$, i.e., $\epsilon_{F}<0$. This is the case if the Fermi energy is below the center of mass of the DOS, which means that there is large spectral weight below the Fermi energy. The tendency toward ferromagnetism in such a situation was already proposed long ago. $^{1-3,18}$

We now consider nearest-neighbor hopping $t_{i j}$ $=-t^{*} / \sqrt{Z}$ and bond-charge interaction $X_{i j}=X t^{*} / \sqrt{Z}$ on several infinite-dimensional lattices. Their densities of states and phase diagram are shown in Fig. 1. The Gaussian DOS of the hypercubic lattice, $N(\epsilon)=\exp \left(-\epsilon^{2} / 2\right) / \sqrt{2 \pi}$, has a parameter $p=1 / \pi$, and the semielliptic DOS of the Bethe lattice, $N(\epsilon)=\sqrt{4-\epsilon^{2}} / 2 \pi$, has $p=32 / 3 \pi^{3}$, and both have $r$ $=0$ due to particle-hole symmetry. Since $p<\frac{4}{3}$ in both cases, the metal-insulator transition at $U_{c}$, drawn as a solid line in

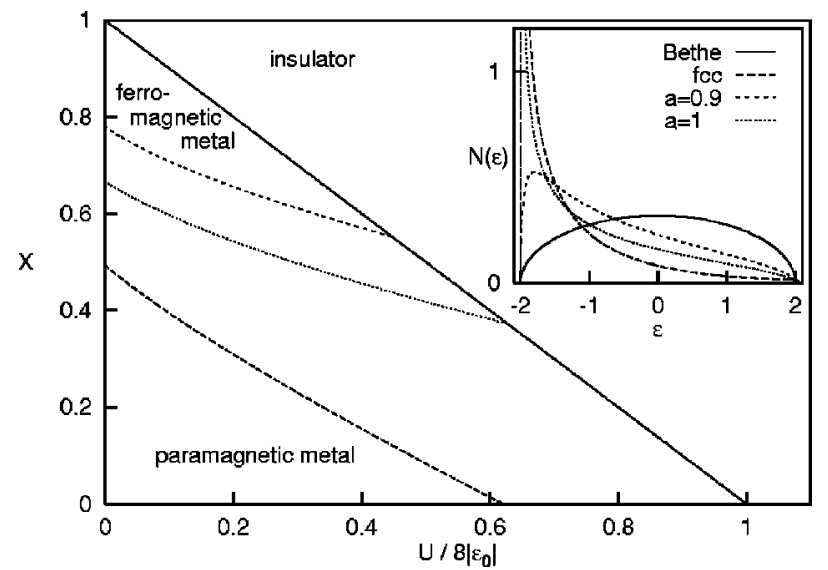

FIG. 1. Phase diagram for the Hubbard model with bond-charge interaction $X$ at half-filling $(n=1)$. The Brinkman-Rice metalinsulator transition takes place for $U_{\mathrm{c}}(X)=8\left|\epsilon_{0}\right|(1-X)$ (solid line). The dashed lines mark the ferromagnetic phase transition for the fcc lattice [Eq. (25)] and for the model DOS of Eq. (26) for $a$ $=1$ and 0.9 , respectively. The inset shows various densities of states, all with unit variance. The lower band edge has been set to the same value for better comparison.

the phase diagram in Fig. 1, will mask the ferromagnetic phase. The variational phase diagram for $\hat{H}_{\text {Hubbard }}$ (i.e., $X$ $=0$ ) on the hypercubic lattice was calculated by Fazekas et al. ${ }^{19}$ who predicted the ferromagnetic and antiferromagnetic phases to coexist as the system phase separates and to preempt the metal-insulator transition. Here we consider only homogeneous ferromagnetic phases, thus allowing the metal-insulator transition to take place, and do not attempt to distinguish between paramagnetic and ferromagnetic insulators at half-filling, which are degenerate in energy $(E=0)$.

A more complicated scenario arises if the lattice system is not particle-hole symmetric, so that the DOS is asymmetric and thus $r \neq 0$. The generalized infinite-dimensional fcc lattice, with hopping scaled as $t_{i j}=-1 / \sqrt{2 D(D-1)}$, has a DOS (Ref. 20)

$$
N(\epsilon)=\frac{\exp (-(1+\sqrt{2} \epsilon) / 2)}{\sqrt{\pi(1+\sqrt{2} \epsilon)}},
$$

showing a square-root singularity at the lower band edge. The Hubbard model [Eq. (1)] on this lattice was studied numerically by Ulmke within dynamical mean-field theory, ${ }^{21}$ who found ferromagnetism at low enough temperatures and band filling. For half-filling one has $\epsilon_{F}=-0.3854, p$ $=1.6157$, and $r=-1.0272$. Thus ferromagnetism occurs in this case already for $X=0$, but the critical $U$ is lowered by the presence of $X>0$. Hence a ferromagnetic phase is found for $U_{\mathrm{fm}}(X)<U<U_{c}(X)$.

Finally, for the class of densities of states with $p<\frac{4}{3}$ ferromagnetism is absent for $X=0$ for all $U$, and is only enabled by switching on the bond-charge interaction $X>0$. It is useful to consider a model DOS, ${ }^{22}$

$$
N(\epsilon)=\frac{1+\sqrt{1-a^{2}}}{2 \pi} \frac{\sqrt{4-\epsilon^{2}}}{2+a \epsilon},
$$




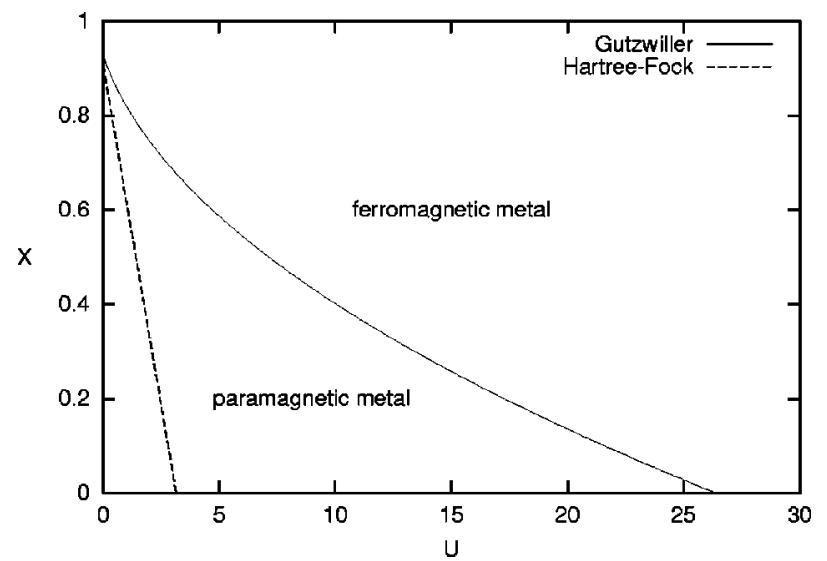

FIG. 2. Phase diagram for the Bethe lattice at density $n=0.9$. The Gutzwiller approximation gives a much smaller region of stability of ferromagnetism than Hartree-Fock theory.

where the tunable parameter $a=-1, \ldots, 1$ determines the distribution of spectral weight: for $a=0$ this DOS reduces to the Bethe DOS, whereas for $a=1$ there is a square-root singularity at the lower band-edge similar to the fcc DOS. For $a=1$ we have $p=1.1353$ and $r=-0.5006$, while for $a$ $=0.9$ the parameters are $p=1.1008$ and $r=-0.2821$. Thus in these cases only a metal-insulator transition is found for $X=0$, but for large enough $X$ a ferromagnetic phase is predicted, as shown in Fig. 1.

Away from half-filling. Since the metal-insulator transition takes place only at half-filling, metallic ferromagnetism occupies a larger part of the phase diagram for $n \neq 1$. Figure 2 shows results for the Bethe lattice with particle density $n$ $=0.9$. Whereas for half-filling the strong-coupling phase was insulating, now there is metallic behavior for all couplings, with ferromagnetism setting in for large $U$ when $X$ is small, and moving to small $U$ when $X$ becomes large. Compared to Hartree-Fock theory the Gutzwiller approximation predicts a much reduced region of stability of ferromagnetism, which is due to the correlated nature of the Gutzwiller wave function. While in Hartree-Fock theory, owing to the lack of correlations, double occupation can be reduced only through a global spin polarization of the system, the Gutzwiller wave function describes a paramagnetic state with reduced double occupation controlled by the variational parameter $g$. Furthermore, the Hartree-Fock prediction of a phase boundary in a range where either $U$ or $X$ is comparable with the hopping amplitude (see Fig. 2), is not consistent with the weakcoupling nature of this approximation, i.e., self-consistent perturbation theory to first order in $U$ and $X$. Hence we expect that the Gutzwiller wave function in general provides a quantitatively better estimate than Hartree-Fock theory.

Figure 3 shows the $U$ vs $n$ phase diagram for the model

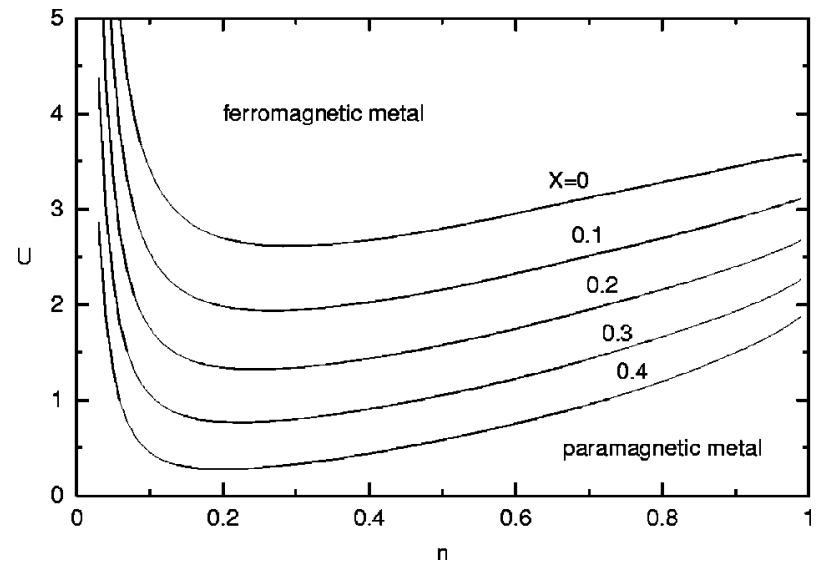

FIG. 3. Phase diagram for the model density of states [Eq. (26)] for $a=0.9$ away from half-filling.

DOS of Eq. (26) with $a=0.9$ for several values of $X$. Already for $X=0$ the Gutzwiller theory predicts a large region of ferromagnetic ground states, in qualitative agreement with numerical results. ${ }^{22}$ The bond-charge interaction again leads to a further stabilization of ferromagnetism.

Conclusion. We found that within Gutzwiller's approach the bond-charge interaction can enhance the instability towards ferromagnetism both at and away from half-filling. This effect is particularly strong when the uncorrelated DOS is asymmetric, and there is large spectral weight below the Fermi energy. This provides further support for the conclusion $^{22,18}$ that such a situation is favorable for ferromagnetism. At half-filling, the presence of the bond-charge interaction leads to a metal-insulator transition at lower values than in the standard Brinkman-Rice scenario, since it tends to immobilize the electrons. Although the Gutzwiller theory can be expected to be reliable only at small to intermediate couplings, it represents a major improvement over Hartree-Fock theory, which, for example, cannot describe a nonmagnetic localization transition.

In conclusion the bond-charge interaction leads to a subtle competition between paramagnetism, ferromagnetism, and localization. Of course, a variational method is not capable of proving the actual stability of a phase. It can only provide estimates for the occurrence of instabilities. Nevertheless, since the Gutzwiller theory treats kinetic and interaction effects nonperturbatively on the same footing, it provides additional insight into the physical mechanism behind these instabilities.

This work was supported in part by the Sonderforschungsbereich 484 of the Deutsche Forschungsgemeinschaft. We would like to thank K. Held for providing us with the Hartree-Fock data of Fig. 2.

\footnotetext{
*Present address: Dept. of Physics, Yale University, P. O. Box 208120, New Haven, CT 06520-8120.

${ }^{1}$ J. Hubbard, Proc. R. Soc. London, Ser. A 276, 238 (1963).

${ }^{2}$ J. Kanamori, Prog. Theor. Phys. 30, 275 (1963).

${ }^{3}$ M.C. Gutzwiller, Phys. Rev. Lett. 10, 159 (1963); Phys. Rev. 134,
}

A923 (1964); 137, A1726 (1965)

${ }^{4}$ For a review, see F. Gebhard, The Mott Metal-Insulator Transition (Springer-Verlag, Berlin, 1997).

${ }^{5}$ J.T. Gammel and D.K. Campbell, Phys. Rev. Lett. 60, C 71 (1988); D.K. Campbell, J.T. Gammel, and E.Y. Loh, Jr., Phys. 
Rev. B 38, 12043 (1988).

${ }^{6}$ J.E. Hirsch, Phys. Rev. B 40, 2354 (1989); 40, 9061 (1989); S. Tang and J.E. Hirsch, ibid. 42, 771 (1990); J.E. Hirsch, ibid. 43, 705 (1991).

${ }^{7}$ Although there is some ambiguity over what is called bondcharge interaction in the literature, we will adopt this name for $X_{i j}$ throughout this paper.

${ }^{8}$ J. Amadon and J.E. Hirsch, Phys. Rev. B 54, 6364 (1996).

${ }^{9}$ A. Schiller, Phys. Rev. B 60, 15660 (1999).

${ }^{10}$ W. Metzner and D. Vollhardt, Phys. Rev. Lett. 62, 324 (1989).

${ }^{11}$ A. Georges, G. Kotliar, W. Krauth, and M. Rozenberg, Rev. Mod. Phys. 68, 13 (1996).

${ }^{12}$ W. Metzner and D. Vollhardt, Phys. Rev. Lett. 59, 121 (1987); Phys. Rev. B 37, 7382 (1988).

${ }^{13}$ W. Metzner, Z. Phys. B: Condens. Matter 77, 253 (1989).

${ }^{14}$ F. Gebhard, Phys. Rev. B 41, 9452 (1990).

${ }^{15}$ D. Vollhardt, Rev. Mod. Phys. 56, 99 (1984).
${ }^{16}$ W.F. Brinkman and T.M. Rice, Phys. Rev. B 2, 4302 (1970).

${ }^{17}$ J. Schlipf, M. Jarrell, P.G.J. van Dongen, N. Blümer, S. Kehrein, Th. Pruschke, and D. Vollhardt, Phys. Rev. Lett. 82, 4890 (1999).

${ }^{18}$ For a recent review of the microscopic conditions favoring metallic ferromagnetism in the one-band and multiband Hubbard models, see D. Vollhardt, N. Blümer, K. Held, M. Kollar, J. Schlipf, M. Ulmke, and J. Wahle, Adv. Solid State Phys. 38, 383 (1999).

${ }^{19}$ P. Fazekas, B. Menge, and E. Müller-Hartmann, Z. Phys. B: Condens. Matter 78, 69 (1990).

${ }^{20}$ E. Müller-Hartmann, in Proceedings of the Fifth Symposium “Physics of Metals," Ustroń, Poland, edited by E. Talik and J. Szade (1991), p. 22.

${ }^{21}$ M. Ulmke, Eur. Phys. J. B 1, 301 (1998).

${ }^{22}$ J. Wahle, N. Blümer, J. Schlipf, K. Held, and D. Vollhardt, Phys. Rev. B 58, 12749 (1998). 